\title{
DESIGN AND OPERATION OF THE CHICAGO AIR SHOWER ARRAY*
}

\author{
L.J Rosenberg, J.W. Cronin, B.E. Fick, K.G. Gibbs, H.A. Krimm \\ N.C. Mascarenhas, T.A. McKay, \\ D. Müller, B.J. Newport, R.A. Ong, M.E. Wiedenbeck
}

The Enrico Fermi Institute, The University of Chicago, Chicago, IL 60637 U.S.A.

K.D. Green, J. Matthews, D. Nitz, D. Sinclair, J.C. van der Velde

Department of Physics, The University of Michigan, Ann Arbor, MI 48109 U.S.A.

\section{ABSTRACT}

The Chicago Air Shower Array (CASA) is a large-area surface scintillator array designed to study PeV sources of cosmic rays. The complete detector will consist of 1089 detector stations, distributed on a square $15 \mathrm{~m}$ grid. We have operated an array of 49 stations for much of the 1989 calender year, an array of 529 stations for much of 1990 , and the balance of the 1089 stations will be operating in early 1991 . This surface array, together with the University of Michigan underground muon detectors (MIA), and the University of Utah atmospheric Cerenkov telescopes and Fly's Eye air fluorescence detector, constitute a uniquely powerful instrument, dubbed the Utah Michigan Chicago (UMC) experiment, for the study of PeV sources. We report here the performance and current status of these detectors.

\section{CASA CONFIGURATION}

CASA is large-area shower array designed to study $\mathrm{PeV}$ sources of cosmic rays, with design goals of good angular resolution, high event rate, and extensive muon detection. Details of CASA can be found elsewhere. ${ }^{1}$ The results from 1989 data are presented in a companion paper. ${ }^{2} \mathrm{CASA}$ is located in Dugway, Utah (USA) at latitude $40.2^{\circ} \mathrm{N}$ and longitude $112.8^{\circ} \mathrm{W}$, at a nominal atmospheric depth of $870 \mathrm{~g} / \mathrm{cm}^{2}$.

A schematic aerial view of the UMC detectors are shown in Fig. 1. Each small dot is one of 1089 CASA stations. Each solid rectangle is one patch of the MIA underground detectors, and each large dot is one of the Utah Cerenkov telescopes. Not shown at the center of the figure is the Fly's Eye II installation. The 49 stations $\left(0.08 \times 10^{5} \mathrm{~m}^{2}\right)$ outlined in the upper left represent the first stage of the CASA array as commissioned in early 1989. The larger outlined region represents the CASA 529 station array $\left(1.1 \times 10^{5} \mathrm{~m}^{2}\right)$ as of early 1990 . The balance of the 1089 station array $\left(2.3 \times 10^{5} \mathrm{~m}^{2}\right)$ will be operating in early 1991 .

An exploded view of one of the CASA stations is shown in Fig. 2. The components of each station are housed within a water-tight two-piece plastic (ABS UV resistant) box. Each box is covered with one radiation length of lead. The critical energy of lead is about $7 \mathrm{MeV}$, and the critical energy of air is about $84 \mathrm{MeV}$. The lead therefore converts some of the photons produced earlier in the shower into $e^{+} e^{-}$pairs, which lowers the detector energy threshold, and sharpens the timing of the shower front. The loading from the lead is carried by the edges of the box and a Styrofoam pillar at the center. A wooden cross member at the bottom of the box separates the interior into four compartments, one for each of the counter assemblies, and supports a plywood mounting surface carrying a circuit board and power supplies. Each counter assembly is composed of one square sheet of acrylic scintillator $1.27 \mathrm{~cm}$ thick and $61 \mathrm{~cm}$ on a side, with a photomultiplier tube glued onto the center face. The phototube and scintillator assembly is housed in a light-tight styrene tray, with a paper honeycomb stiffener mounted to the bottom. Four such counters are placed in each station as shown in the figure.

\footnotetext{
*Proc. Int. Conf. on High Energy Gamma-Ray Astronomy, Oct. 2-5, 1990, Ann Arbor, MI.
} 


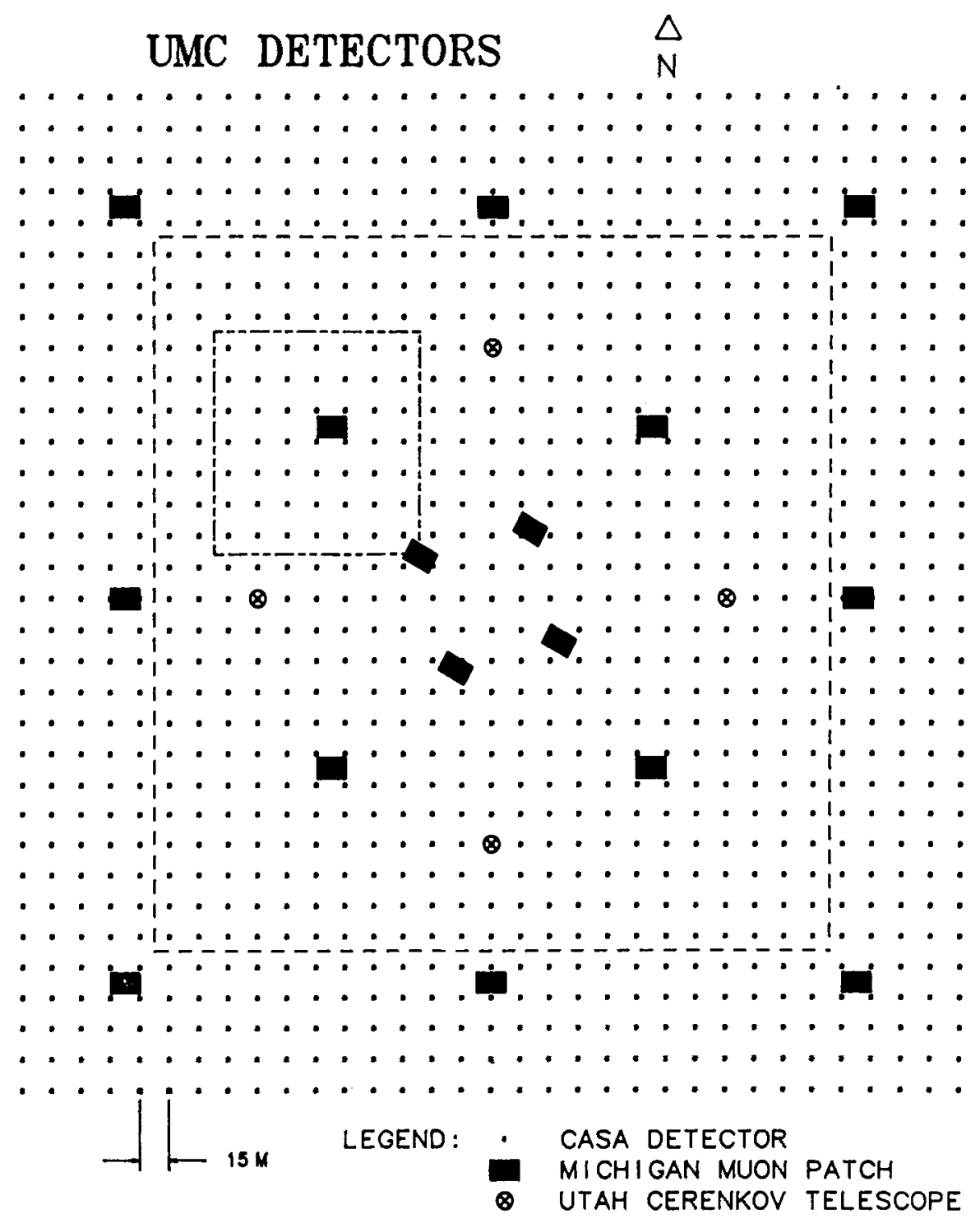

Figure 1. A schematic aerial view of the UMC detectors. Each small dot is one of 1089 CASA stations. Each solid rectangle is one patch of the MIA underground detectors, and each large dot is one of the Utah Cerenkov telescopes. Not shown at the center of the figure is the Fly's Eye II installation. The 49 stations $\left(0.08 \times 10^{5} \mathrm{~m}^{2}\right)$ outlined in the upper left represent the first stage of the CASA array as commissioned in early 1989 . The larger outlined region represents the CASA 529 station array $\left(1.1 \times 10^{5} \mathrm{~m}^{2}\right)$ as of early 1990 . The balance of the 1089 station array $\left(2.3 \times 10^{5} \mathrm{~m}^{2}\right)$ will be operating in early 1991 . 


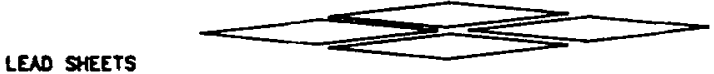

LEAD SHEETS
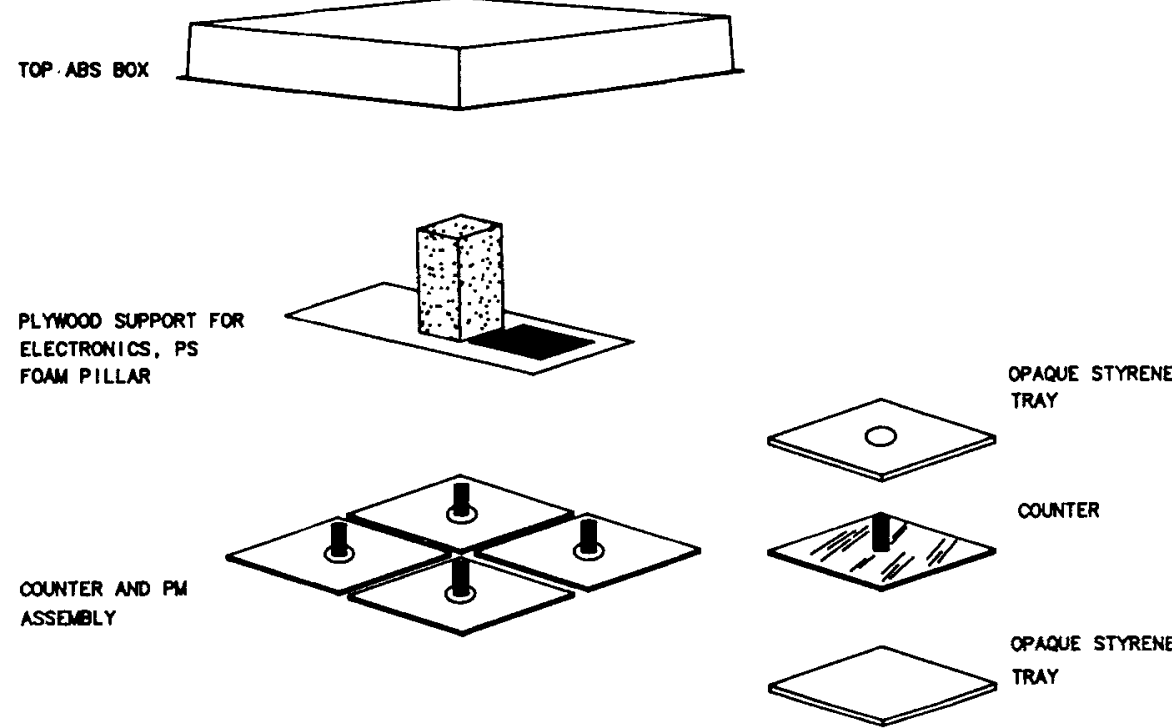

COUNTER ND PM
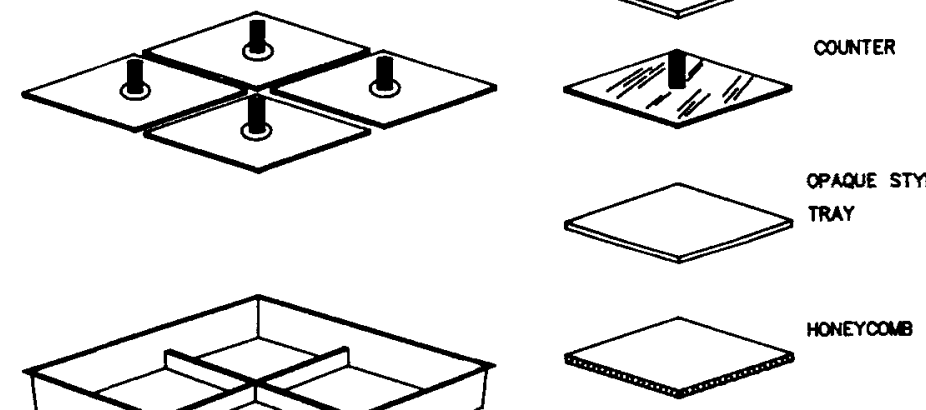

BOX: ABS \& UN IAHIBITOR

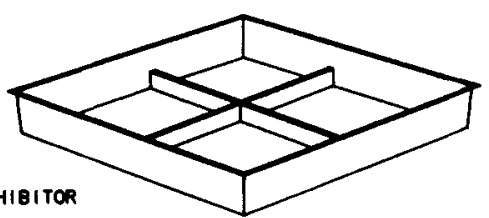

DOTTOM WITH STIFFENER

EXPLODED VIEW

OF COUNTER AN PM ASSEMaLY

Figure 2. An exploded view of one of the CASA stations. 


\section{The Chicago Air Shower Array}

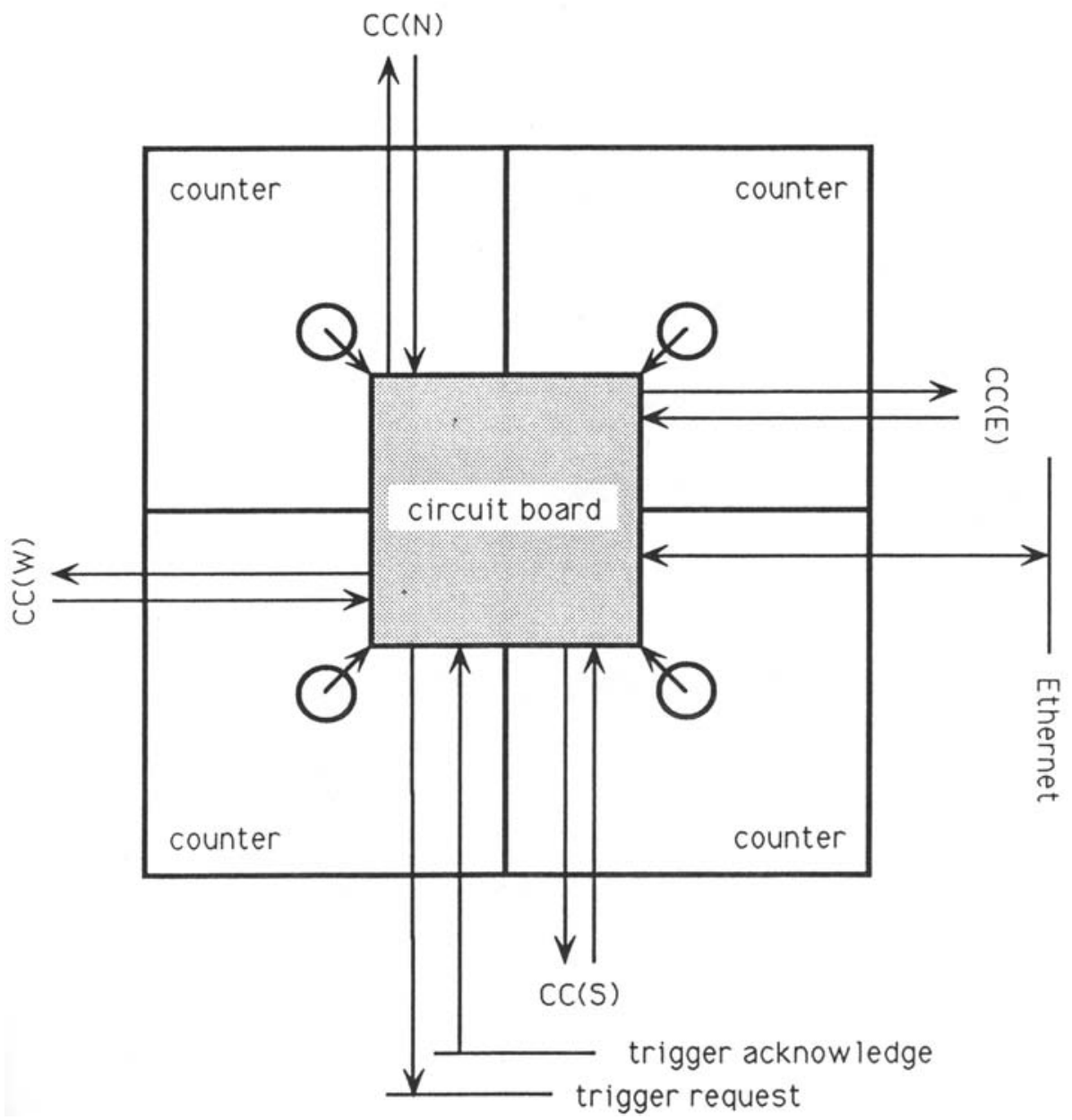

Figure 3. A simplified sketch of the electrical connections to and from a CASA station circuit board. The cross connections to and from adjacent stations are denoted $\mathrm{CC}(\mathrm{N})$, $\mathrm{CC}(\mathrm{E}), \mathrm{CC}(\mathrm{S})$, and $\mathrm{CC}(\mathrm{W})$. 


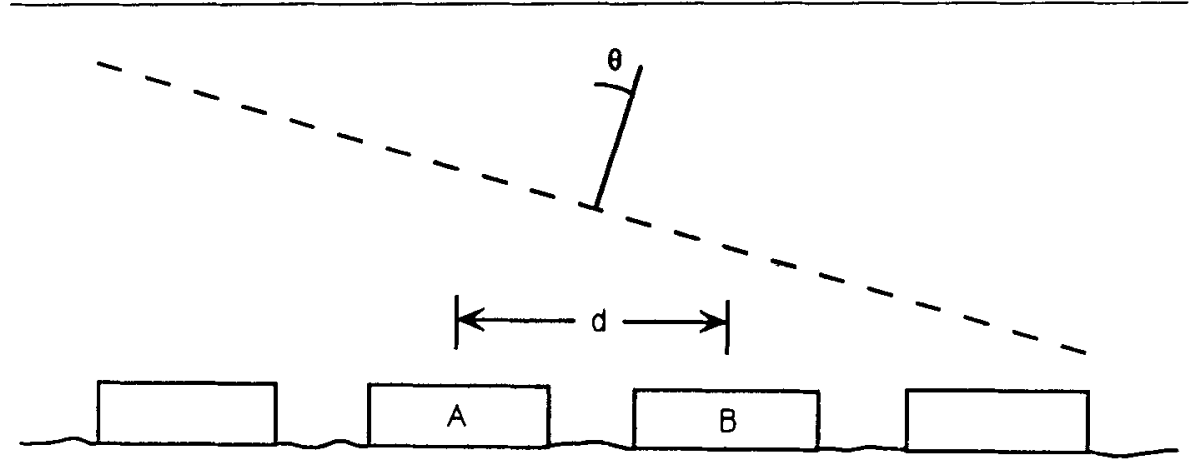

Figure 4. A shower incident on the CASA array illustrating the geometry referred to in the text.

A simplified sketch of the connections to a CASA circuit board is shown in Fig. 3. The circuit board is represented by the square at the center of the figure. The four counters are represented by the other four squares, with the phototubes shown as the circles. There are two cables running from the circuit board to each phototube base. One of these cables carries high voltage, derived from a programmable DC to HVDC converter module mounted to the circuit board. The other cable carries the anode signal to an ADC and TDC channel on the circuit board. A timing signal derived from the phototube pulses is split four ways and sent to each of the adjacent stations on the square grid along twisted pair cables, as shown by the four arrows leaving the edge of the board, and the timing signals sent by the four nearest. neighbors are similarly received on four twisted pair cables. These signals which are sent to the nearest stations are called cross connections. There are in addition three bussed coaxial cable connections to each station. The stations use one of these, the trigger request cable, to inject a current pulse when a station finds that a minimum number of counters fire within a 20 ns window. When enough stations inject current into the trigger request line within a. 10 r $1 \mathrm{~s}$ window (likely due to an air shower landing on the array), a pulse is applied to the trigger acknowledge coaxial cable; this notifies the CASA stations that there is an array trigger. At the receipt of this trigger acknowledge signal, stations digitize the data and save it in a local buffer. The buffer is periodically (approximately every $30 \mathrm{~s}$ ) uploaded over the final cable, an Ethernet connection, to a data acquisition VAX. Not shown is the connection of each station to the $\mathrm{AC}$ mains power. Note that this cabling scheme results in only three signal lines feeding centralized electronics: trigger request, trigger acknowledge, and Ethernet. Problems relating to maintaining signal quality and leading edge timing over long cable runs are therefore eliminated.

\section{CASA TRIGGER AND OPERATION}

This method of utilizing cross connections allows the CASA experiment to reconstruct the shower source direction without stringing cables into centralized data acquisition electronics. Consider the simple shower geometry illustrated in Fig. 4. In this case, a shower coming from zenith angle $\theta$ is incident on the CASA array. Let us concentrate on just two of the boxes $A$ and $B$ separated by a distance $d$. The shower direction cosine is given by $(d / c) \sin \theta=T_{A}-T_{B}$ with $T_{A}$ the absolute time when the shower front arrives at station $A$, and $T_{B}$ the absolute time when the shower front arrives at station $B$. The TDC's in station $A$ record a time difference $t_{A}=T_{A}-T_{A}^{0}$, with $T_{A}^{0}$ an arbitrary start time. Similarly, station $B$ records a time difference $t_{B}=T_{B}-T_{B}^{0}$, with $T_{B}^{0}$ another arbitrary start time. An unusual feature of the CASA design is that each station records cross times from its neighbors. For example, one of these cross times is given by $t_{A, B}=T_{A}^{0}-T_{B}^{0}$, for the case of the cross time recorded by station $A$ from the timing 


\section{The Chicago Air Shower Array}

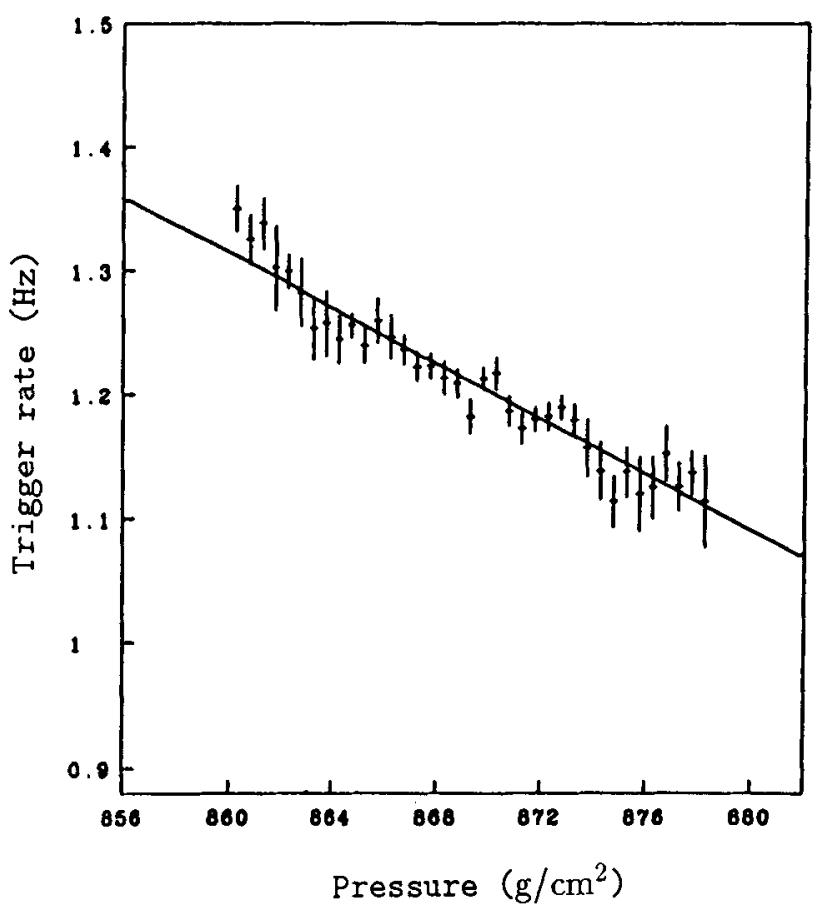

Figure 5. The CASA (49 station array) trigger rate as a function of atmospheric pressure.

signal sent out by station $B$. We can show that $T_{A}-T_{B}=t_{A}-t_{B}+(1 / 2)\left\{t_{A, B}-t_{B, A}\right\}$. In other words, these arbitrary start times cancel in the determination of the shower arrival direction.

Most of the time, a station is idle, that is, a station is waiting for its counters to be struck. If a sufficient number of a station's counters are struck within $20 \mathrm{~ns}$ (the number is programmable - the nominal requirement is at least two of four counters), then the station is said to be "alerted." An alerted station activates its pulse height, local TDC, and cross connection TDC sample and holds. A stronger condition occurs when a station is "triggered" (this condition is also programmable - the nominal requirement is at least three of four counters). A triggered station undergoes all the activity for the alerted case, but in addition injects a current pulse into the trigger request line. Should three or more of these pulses from different stations arrive at a central VAX within approximately $5 \mu \mathrm{s}$, then a signal is asserted on the trigger ackinowledge line. This signal is recognized by the alerted and triggered stations as the command to digitize the voltages stored on the sample and holds, and save the digitized data in a local buffer. The alerted and triggered station are dead for this digitization time of approximately $400 \mu \mathrm{s}$. On the other hand, should a pulse not appear on the trigger acknowledge line some $10 \mu$ s after a station is alerted or triggered, then the station resets itself and clears the stored charge on the sample and holds.

The array trigger rate depends on pressure. This dependence is shown in Fig. 5 for data from the 49 station array. Since the CASA array is well below shower maximum for most events, the trigger rate decreases with increasing atmospheric overburden. The nominal trigger rates are $1.2 \mathrm{~Hz}$ for the 49 station array, $14.0 \mathrm{~Hz}$ from the 529 station array, and $28.0 \mathrm{~Hz}$ expected from the 1089 station array. 

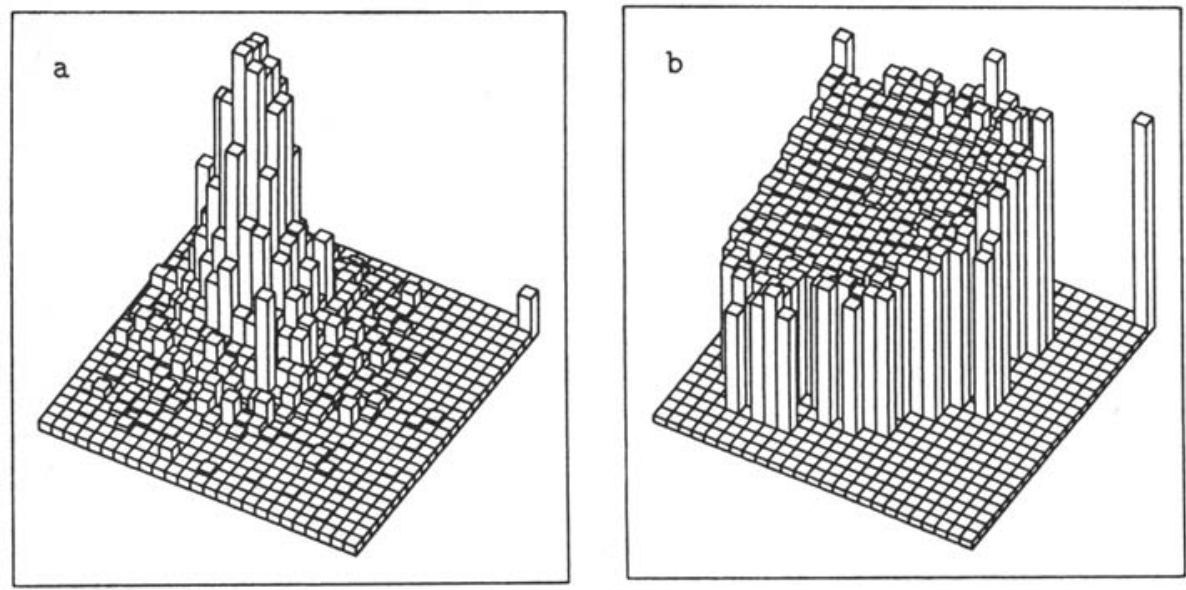

Figure 6. A large shower from the CASA 529 station data. Each square represents one of the CASA stations. (a) The height represents the number of particles striking each station. The square to the far right is the scale representing 10 particles. (b) The height represents the relative arrival time of the shower front. The square at the far right is the scale representing $200 \mathrm{~ns}$.

The data acquisition readout is controlled by two VAX's. One of them receives triggers through CAMAC from the CASA, muon, Cerenkov, and Fly's Eye systems, reads out the nonCASA data, and sends the data to the other VAX over a network Ethernet connection. This other VAX reads out data from the CASA stations over the CASA Ethernet, merges the data from both VAX's into one output data stream, and periodically logs the combined data to $8 \mathrm{~mm}$ tape. We log approximately $10^{6}$ events/day from the 529 station array, and expect to log $2 \times 10^{6}$ events/day from the 1089 station array. In terms of data rates, this is approximately 1 GByte/day and 2 GByte/day respectively.

For each CASA trigger, we determine the number of particles incident on each CASA station as the pulse height less pedestal in units of minimum ionization. This number of sampled particles is fitted to an NKG function with age fixed at 1.28. This procedure yields the shower size (that is, the number of particles striking the ground). The internal and cross time TDC. counts in each station are converted into times, referenced to a crystal time base in each station. These times yield an ensemble of direction cosines pointing to the shower arrival direction. The reconstructed shower arrival direction is the average of the direction cosines, weighted with the number of particles participating in each direction cosine link.

A rather large shower from the CASA 529 station data is shown in Fig. 6a. Each square represents one of the CASA stations, and the height represents the number of particles striking each station. The square to the far right is the scale representing 10 particles. Note the prominent core at the center of the shower. The companion Fig. $6 \mathrm{~b}$ shows the timing differences from the same event. The height represents the relative arrival time of the shower front from station to station, and the square at the far right is the scale representing $200 \mathrm{~ns}$. The timing: front is flat, especially near the core, mirroring the flatness of the incident shower front. The shower arrival direction is normal to the plane. 


\section{The Chicago Air Shower Array}
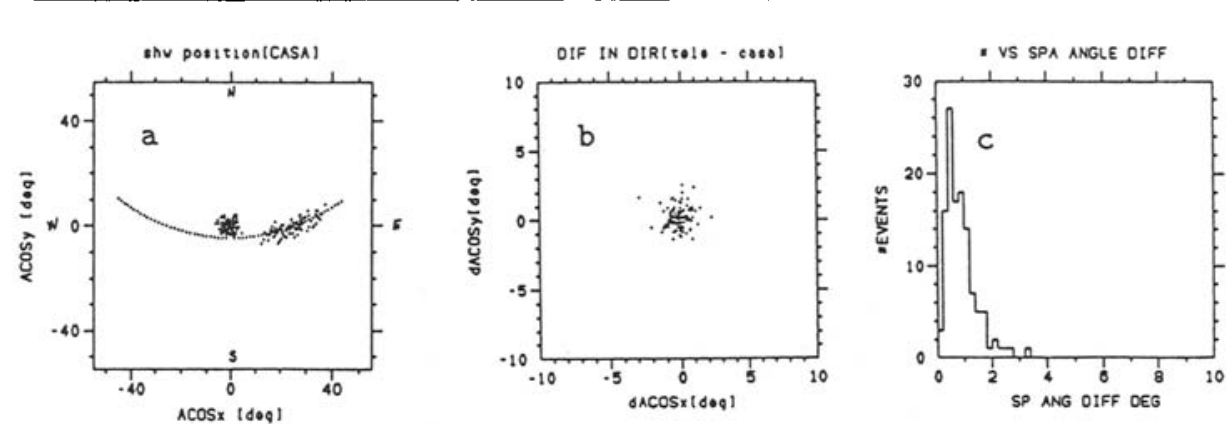

Figure 7. Cerenkov telescope study of accuracy and resolution of CASA. (a) The arrival direction of those CASA events which had coincident telescope events. (b) The difference in directions between these CASA events and the same events as seen by the telescopes. (c) The distribution of space angle differences between the CASA and telescope determination of the arrival direction.

\section{ANGULAR RESOLUTION AND ACCURACY}

A powerful method to determine the accuracy and resolution of the CASA array is to compare shower directions as determined by CASA with shower directions as determined by the Cerenkov telescopes. Since the resolution of the telescopes is better than the CASA resolution, this comparison is largely a measure of the CASA resolution. As an example of this technique, we describe the results of one night of study during which we pointed the telescope directly towards the zenith for roughly half of the night, and tracked Her X-1 for the remainder of the evening. In Fig. 7a, we show the arrival direction of those CASA events which had coincident telescope events. The center of the figure is straight up. The ordinate is the North-South direction in degrees, and the abscissa is the East-West direction in degrees. The 138 events bunched at the zenith are from the earlier part of the night, while the 92 events which lie along the dotted track of Her X-1 are from later in the night. The difference in directions between these CASA events and the same events as seen by the telescopes is shown in Fig. 7b, where the ordinate is the angular difference in the North-South direction in degrees, and the abscissa is the angular difference in the East-West direction in degrees. The center of the figure is where events lie when CASA and the telescopes yield identical shower arrival directions. Note that there is no bias in the accuracy of the CASA determination of the arrival direction; the events are bunched near the center of the figure with no obvious pull in any direction. Finally, in Fig. 7c, we show the distribution of space angle differences between the CASA and telescope determination of the arrival direction. Observe that some $63 \%$ of the CASA events lie within about $1^{\circ}$ of the direction determined by the telescopes.

Another way we measure the angular resolution is to use the "split detector" technique where half of the counters in a station are assigned to one sub-detector, and the other counters in a station are assigned to the other sub-detector. Each sub-detector finds the incident shower direction independently, and the resolution of the whole CASA array is related to the difference between these measurements. In Fig. 8 we show the angular resolution for the whole CASA 


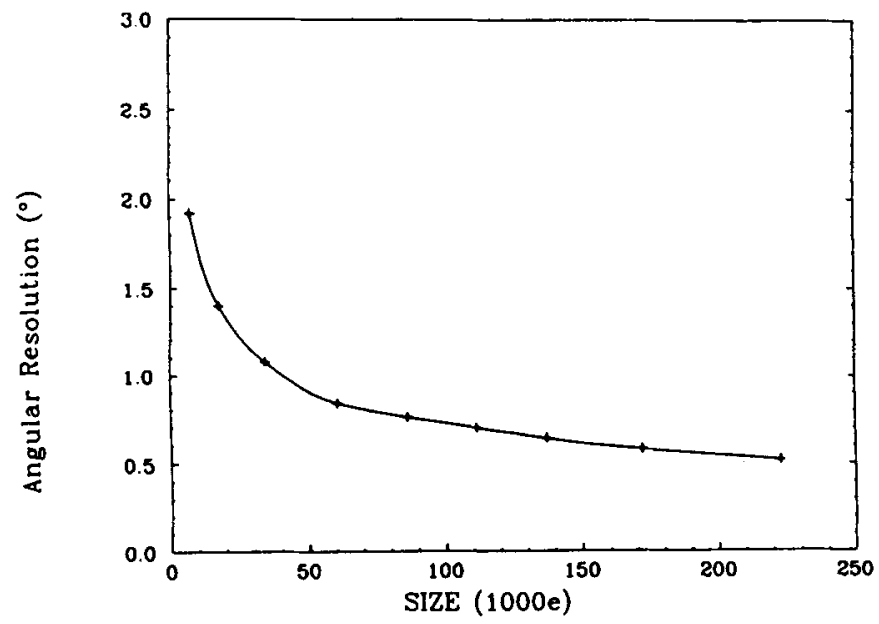

Figure 8 . The angular resolution (the half angle cone containing $63 \%$ of events from a point source) from the split detector study of angular resolution.

array as a function of the shower size from 529 station data, where the angular resolution is defined as the half angle cone containing $63 \%$ of the events. The angular resolution is about $1^{\circ}$ at the mean CASA shower size of 25000 particles, and improves as the showers get larger.

\section{THE MUON SYSTEM}

The University of Michigan has installed muon detectors ${ }^{3}$ below the CASA surface array. Each detector element is a $1.3 \mathrm{~m} \times 1.9 \mathrm{~m}$ sheet of scintillator, fitted with a 5 inch hemispherical phototube in a milled opening. Sixty four such counters are assembled into a patch, and buried $3 \mathrm{~m}$ below the surface. One of these patches is illustrated in Fig. 9. There are sixteen such patches, distributed as shown in Fig. 1. The high voltage and signal for each counter is carried on one cable, and decoupled in the data acquisition area. The signals are discriminated and fed into CAMAC TDC's. The common stop is derived from the CASA shower timing.

A simplified description of the muon reconstruction follows. We first determine which of the CASA stations generated the current pulse which references the muon TDC stops; the cable delay from this station to the muon TDC's is subtracted. We then propagate the shower front, as determined by CASA, to each muon counter, accounting for the cable delays from each muon counter to its corresponding TDC. We define a muon as a TDC stop within $\pm 100 \mathrm{~ns}$ of the shower front passing through a counter. The distribution of muon times relative to the crossing of the shower front is illustrated in Fig. 10 from 529 station data, where the \pm 100 ns window is seen to be a many $\sigma$ cut.

In Fig. 11a we show the average number of in time muons as a function of the number of alerted stations (this roughly corresponds to shower size) from 529 station data taken with eight muon patches in operation. We see that the number of muons increases with shower size. We now consider the average number of out of time muons versus the number of alerted stations, as shown in Fig. 11b. This flat constant background of 10 muons represents the accidental rate into the muon TDC gate of $4 \mu \mathrm{s}$. The contamination of accidentals from eight patches into the $\pm 100 \mathrm{~ns}$ window is therefore 0.5 muons per event. 


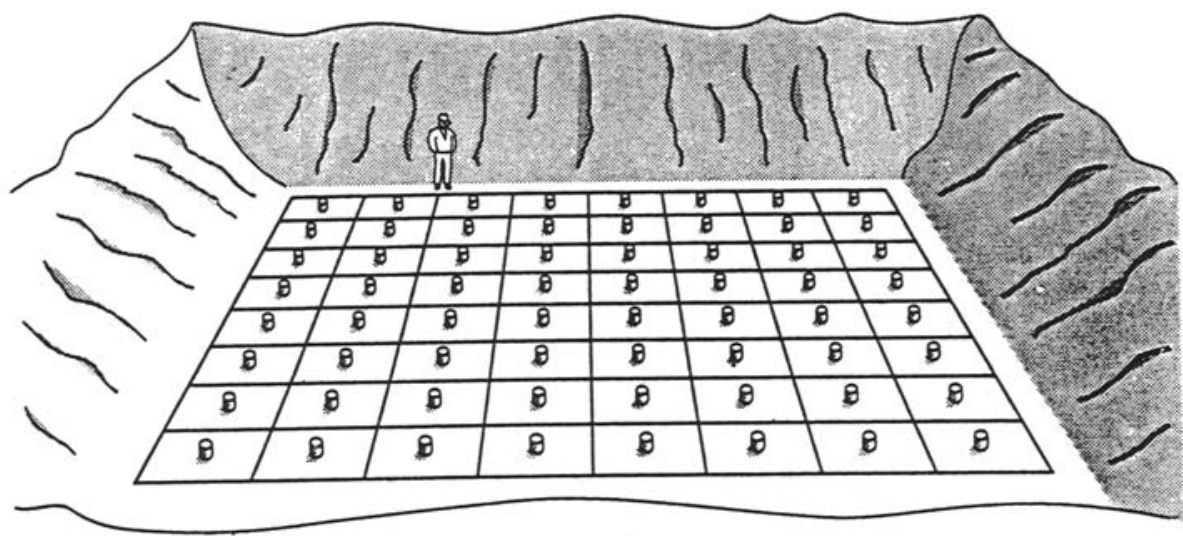

Figure 9. Sketch of one of sixteen muon patches before it is covered with $3 \mathrm{~m}$ of dirt.

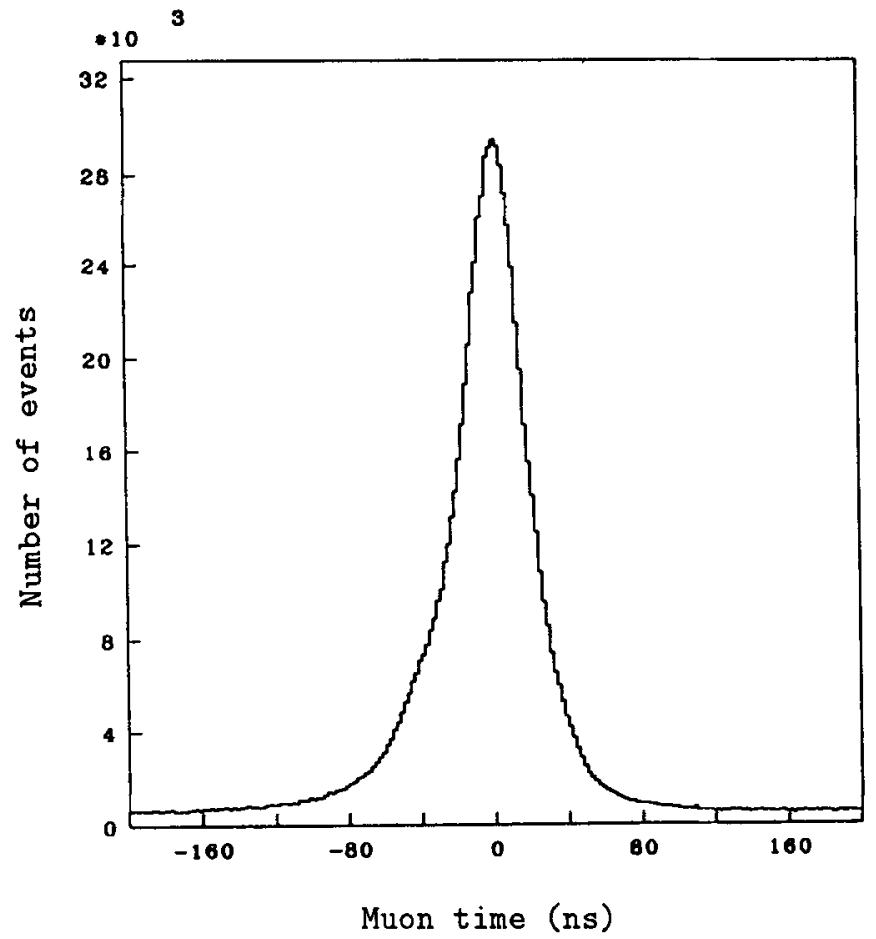

Figure 10. Spread of muon times relative to the time the shower front crosses the muon counter. 

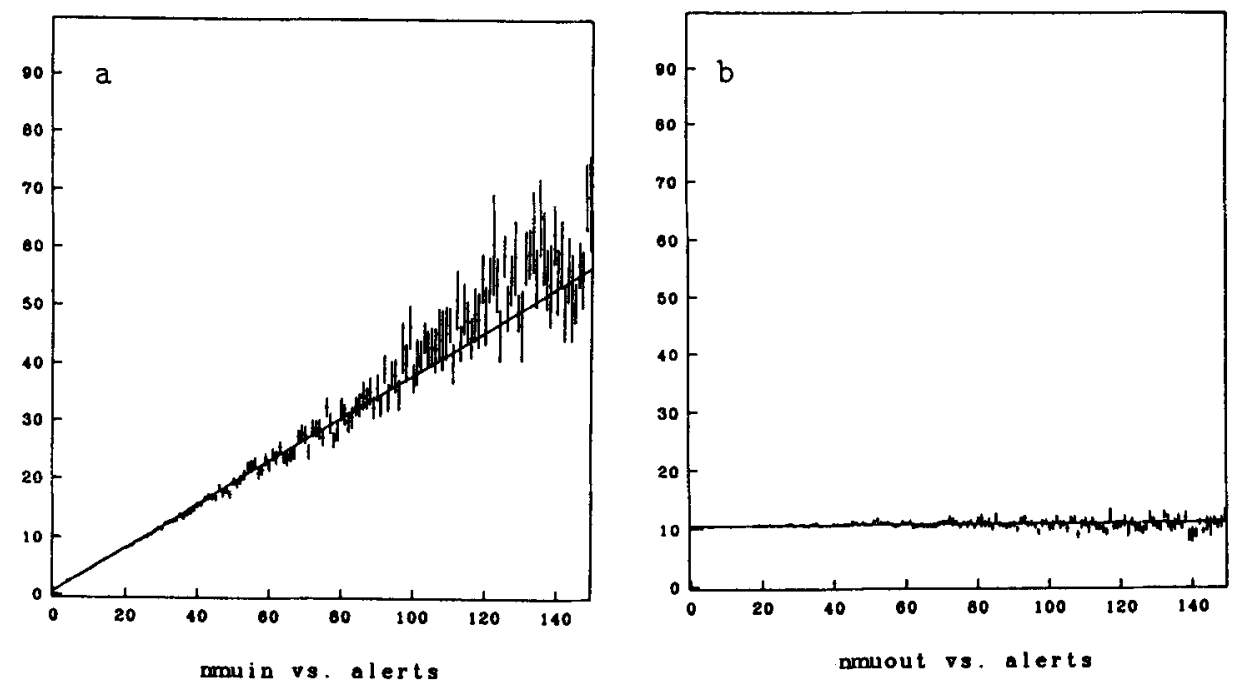

Figure 11. (a) Average number of in time muons versus number of alerted stations. (b) Average number of out of time muons versus the number of alerted stations.

\section{CONCLUSIONS}

The CASA experiment has grown from a 3 station prototype in 1987, through a 49 station array in 1989 , a 529 station array in 1990 , and we are well on the way towards completing the 1089 station array in early 1991. The shower direction is determined accurately, and the angular resolution approaches $0.5^{\circ}$ for sufficiently large showers. CASA is now more sensitive and has more muon coverage than any other array designed to study $\mathrm{PeV}$ sources of cosmic rays.

We gratefully acknowledge the help and support of the members of the University of Utah Fly's Eye Collaboration and of Colonel Van Prooyen and the staff of the Dugway Proving Ground. This work is supported in part by the National Science Foundation and the U.S Department of Energy.

\section{REFERENCES}

1. K.G. Gibbs et al., Proc. 20th Intl. Cosmic Ray Conf., Adelaide, Australia 4 (1990) 306;

R.A. Ong et al., Proc. 20th Intl. Cosmic Ray Conf., Adelaide, Australia 4 (1990) 310;

B.J. Newport et al., Proc. Vulcano Workshop, Vulcano, Italy (1990);

R.A. Ong, Nucl. Phys. B (Proc. Suppl) 14A (1990) 273;

L.J Rosenberg, in Particle Astrophysics: Forefront Experimental Issues, ed. E. Nolman, World Scientific (1989) 257;

K.G. Gibbs, Nucl. Instr. Meth., A264 (1988) 67;

L.J Rosenberg, Proc. Ultra-High Energy Cosmic Ray Workshop, Univ. of Utah and Bartol Research Foundation (1987) 155.

2. H.A. Krimm et al., This conference.

3. D. Sinclair, Nucl. Instr. Meth., A278 (1989) 583. 